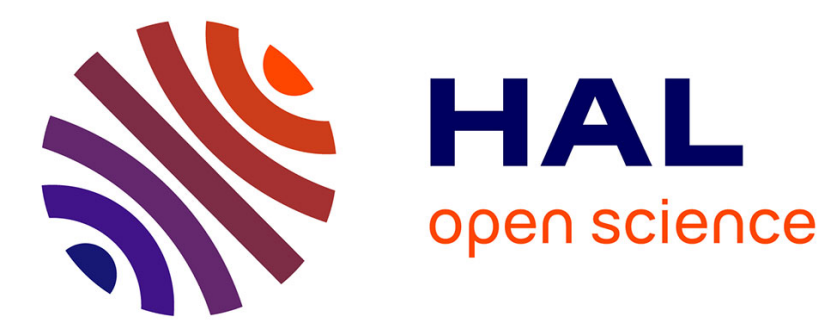

\title{
About the warrants of computer-based knowledge
}

Anouk Barberousse, Marion Vorms

\section{To cite this version:}

Anouk Barberousse, Marion Vorms. About the warrants of computer-based knowledge. Synthese, 2014, pp.20. 10.1007/s11229-014-0482-6 . halshs-01058659

\section{HAL Id: halshs-01058659 \\ https://shs.hal.science/halshs-01058659}

Submitted on 2 Sep 2021

HAL is a multi-disciplinary open access archive for the deposit and dissemination of scientific research documents, whether they are published or not. The documents may come from teaching and research institutions in France or abroad, or from public or private research centers.
L'archive ouverte pluridisciplinaire HAL, est destinée au dépôt et à la diffusion de documents scientifiques de niveau recherche, publiés ou non, émanant des établissements d'enseignement et de recherche français ou étrangers, des laboratoires publics ou privés. 


\title{
About the warrants of computer-based empirical knowledge
}

\section{Anouk Barberousse \& Marion Vorms}

\begin{abstract}
Computer simulations are widely used in current scientific practice, as a tool to obtain information about various phenomena. Scientists accordingly rely on the outputs of computer simulations to make statements about the empirical world. In that sense, simulations seem to enable scientists to acquire empirical knowledge. The aim of this paper is to assess whether computer simulations actually allow for the production of empirical knowledge, and how. It provides an epistemological analysis of present-day empirical science, to which the traditional epistemological categories cannot apply in any simple way. Our strategy consists in acknowledging the complexity of scientific practice, and trying to assess its rationality. Hence, while we are careful not to abstract away from the details of scientific practice, our approach is not strictly descriptive: our goal is to state in what conditions empirical science can rely on computer simulations. In order to do so, we need to adopt a renewed epistemological framework, whose categories would enable us to give a finer-grained, and better-fitted analysis of the rationality of scientific practice.
\end{abstract}

\section{Key words}

computer simulation, empirical knowledge, entitlement, justification, warrant, expertise 


\section{Introduction}

Computer simulations are widely used in current scientific practice, as a tool to obtain information about various phenomena. Scientists accordingly rely on the outputs of computer simulations to make statements about the empirical world. In that sense, simulations seem to enable scientists to acquire empirical knowledge. The aim of this paper is to assess whether computer simulations actually allow for the production of empirical knowledge, and how.

Traditionally, an empirical statement is considered as justified when it is obtained either $i$. through direct perceptual experience, or ii. through rational deduction (or induction) from premisses obtained by direct perceptual experience. However, most of present-day empirical science, and particularly computer-based science, is done in a way to which this conception of justification does not apply in any simple way. If one sticks to the traditional epistemological standards, two main problems arise, which put into question the rationality of the production of empirical knowledge through computer simulations.

(P1) Failure of content-preservation. Firstly, in contrast with the deductive process of a calculation made by hand, the content of the premisses (i.e., of the theoretical model and input data in use in the simulation) is not strictly preserved throughout the computational process of the simulation. As a matter of fact, the original equations have to be discretized and a number of idealizations and additional hypotheses have to be introduced in order to build up the algorithm allowing the computer to calculate the (substitutes of) the original model's equations. Hence, the original, empirical content of the premisses is modified throughout the computational process. By "empirical content of the premisses", we mean what the theoretical model, together with input data, say about the investigated system in the world.

(P2) Loss of epistemic control. Second, human agents cannot follow the successive steps of the computational process. As a consequence, scientists cannot achieve the epistemic 
control that seems necessary for them to be fully justified in accepting the results. They would be so if they could produce, or at least master and assess, an explicit series of well-controlled deductions from the premisses to the results. However, as they cannot access the detailed rationale for the succession of individual steps within the computational process, they cannot reconstruct any deductive argument they could follow and control.

Acknowledging these two problems, one could simply deny that simulations can legitimately help us achieve any empirical knowledge about systems of interest to the scientific community. But this seems at odds with scientific practice. An alternative option, which we endorse, is to explore to what extent, and in what conditions, the practice of relying on computers as a mean to achieve empirical knowledge is rationally warranted. Our starting point is to acknowledge the complexity of scientific practice, whose rationality we then try to assess. Hence, while we are careful not to abstract away from the details of scientific practice, our approach is not strictly descriptive: our goal is to state in what conditions empirical science can rely on computer simulations. In order to do so, we need to adopt a renewed epistemological framework, whose categories would enable us to give a finer-grained, and better-fitted analysis of the rationality of scientific practice.

Some aspects of $(\mathrm{P} 1)$ have been explored in a series of papers comparing computer simulation and experimentation (AUTHOR et al., 2009; Frigg and Reiss, 2009; Hartmann, 1996; Humphreys, 2004, 2009; Lenhard, 2007; Morrison, 2009; Parker, 2008a, 2008b, 2009; Rohrlich, 1991; Winsberg, 1999, 2003, 2009). The main question tackled by these papers is the following one: Where, on the methodological map (that is, where, between theory exploration and experiment) do computer simulations lie? However, when it comes to assessing the justification of computer-based empirical knowledge, hence the rationality of the epistemic practice of relying on the outputs of computer simulations to achieve empirical 
knowledge, answering this question is not enough ${ }^{1}$. A complementary, epistemological, approach is necessary, which consists in focusing on the reasons scientists have to trust the simulations outputs as bearing on the empirical world, if there are any. We put forward this epistemological, agent-centered, approach. Endorsing Eric Winsberg's view that "the epistemology of simulation is [...] the study of the means by which we sanction belief in the outcome of simulation studies, despite their motley methodology" (1999), we try to find out what are the means by which belief-sanctioning is obtained.

Our goal is thus to answer the following questions: How is the empirical knowledge drawn from computer simulations warranted? To what extent, and how, are scientists justified in making statements about the systems they are interested in on the basis of computer simulations' outputs? In section 2, we discuss the traditional epistemological categories and their contemporary extension by Tyler Burge $(1993,1998)$ in his analysis of computerassisted mathematical proofs. This preliminary analysis will offer us the epistemological tools with which we will then tackle (P1) and (P2) respectively. In section 3, we address the problem of the failure of content-preservation in computer simulations, and explore the conditions for a justification of their empirical content. In section 4 , we assess whether, and to what extent, individual agents are warranted in accepting the outputs of computer simulations even though they cannot control the details of their production. We clarify in which sense empirical knowledge drawn from computer simulations is warranted.

\section{Setting the epistemological landscape}

\footnotetext{
${ }^{1}$ We acknowledge that there is an important literature, both in engineering and in the philosophy of science (cf. for instance Winsberg 2003, 2006; Parker 2008a; Lloyd 2010) analyzing various methods, like "Verification \& Validation" methods, helping simulation users to control and assess the validity of the outputs. In order to improve the justificational part of the assessment task with respect to simulation outputs, these methods aim at making control procedures explicit and accessible. In this paper, our goal is rather to uncover aspects of warrant that can hardly be made explicit (see section 4). This is the reason why we exclude "Verification \& Validation" methods from our analysis. We thank an anonymous reviewer for pointing out this aspect of the discussion.
} 
Computers are used by scientists in both empirical science, and mathematics. Although our object is to clarify the warrants of computer-based empirical knowledge, we temporarily ignore, in this section, the question of the empirical content of computer simulations, and (P1). We will focus on (P2), namely on the lack of full epistemic control upon the computational process, which is also an important feature of computer-based mathematical knowledge. Computer simulation users face both (P1) and (P2); but studying (P2) in isolation from (P1) will enable us $i$. to highlight the specificities of (P2) in a simpler case, and $i i$. to settle the epistemological framework that will serve us, later on in the paper, to study the effects of (P1) on (P2) in the building of empirical knowledge. So our main object in this section are the consequences of (P2) on the nature and justification of (any kind of) information acquired through computers: Is it to be called "knowledge"? Is its content somehow altered by its having been achieved through a computational process human agents do not fully control?

We first (2.1) clarify why the loss of epistemic control might be a threat for the justification of computer-based knowledge, by discussing some traditional epistemological categories. We then (2.2) enlarge the epistemological framework to fit some everyday epistemic practices that are already suspect from the perspective of the categories presented in 2.1, namely reliance on memory and testimony, drawing on Burge's $(1993,1998)$ analyses. This extension of our epistemological framework can also apply to computer-assisted mathematical proofs. We present this step in section 2.3 as a first building block of our analysis of the epistemological specificities of the use of computer simulations.

2.1 Traditional epistemology: sources and warrants of a priori and empirical knowledge 
Traditionally, two types of knowledge are distinguished: empirical knowledge, which bears upon the world, and a priori knowledge, which bears upon logic and mathematics. It is generally acknowledged that what makes a piece of knowledge a priori or empirical is the nature of the justification on which it rests. Hence, as Burge (1993, 458, 1998, 3) highlights, "a priori" and "empirical" apply primarily to warrants, and to knowledge only by derivation. Following Burge, we take an epistemic warrant to be what "rationally supports [...] a propositional attitude or cognitive practice, and [...] constitutes an epistemic right to it" (Burge, 1993, 458). True belief needs to be warranted to count as knowledge. Warrants can come under a variety of ways, of which full-fledged justification arguments, namely arguments consisting in well-controlled inferences from premisses to conclusion, and which agents can explicitly assess, are only one kind. A warrant is a priori "if its justificational force is in no way constituted or enhanced by reference to or reliance on the specifics of some range of sense experiences or perceptual beliefs.” (Burge, 1993, 458) A belief counts as a priori knowledge if it is fully justified by a priori warrants, that is, if no element of empirical origin enters into its justification in any essential way (although it might be psychologically indispensable to rely on some empirical support). On the other hand, a piece of knowledge is empirical if its justification requires reference to some range of sense experience. For instance, a warrant (among others) for my belief that it is raining outside is my present auditory experience of the noise of the rain falling over the roof. To be sure, a priori elements (at least elementary logical rules) may enter into the justification of empirical knowledge. But what makes it empirical is the fact that elements of perceptual experience enter in a non dispensable way into its justification.

One traditionally distinguishes between three main sources of knowledge: (i) observation, (ii) deductions from logical or mathematical axioms that are known a priori, and (iii) deductions or inductions from observations. Epistemology traditionally favors sources (i) 
and (ii), because in these epistemic situations, one immediately knows that one possesses genuine knowledge, when one does. Empirical knowledge obtained by visual contact is considered as more justified than knowledge obtained from any kind of reasoning from observational premises, because in the former case we have some immediate access to the object of our belief. The conclusions of chains of mathematical reasoning, of which each step is by itself (immediately) evident, are another touchstone for genuine knowledge, as emphasized by Descartes (1628). Cases of immediate evidence (either intellectual or sensible), namely cases in which the acquisition of information is unmediated by any potential source of error, such as memory, are thus epistemologically privileged.

From this traditional perspective, reliance on computer in order to gain knowledge is obviously suspect. Agents' loss of epistemic control upon the computational process seems to place them far away from any situation of immediate evidence. It is worth noting that, more generally, our epistemic lives rely on many other practices that clearly infringe the epistemological standards described above. First, we heavily rely on memory, both in (i) empirical and (ii) a priori knowledge-acquisition. Indeed, (i) most of our everyday reasoning processes are partially based on stored observations, and (ii) when a long chain of deductions is involved, logical and mathematical reasoning may also be based on stored a priori premises (i.e., logical or mathematical premises, which we remember are true, without their being immediately evident to us). Second, we have learnt the major part of what we know about the world through interlocution (testimony), rather than from direct observation. To what extent is reliance on memory, and on testimony, rational? In what conditions can we consider the conclusions of memory (and testimony-)based reasoning processes justified, and thus legitimately consider them as knowledge? Answering these questions will allow us to tackle computer-assisted epistemic practices from section 2.3 onward. 


\subsection{Everyday epistemological practices}

Memory and testimony seem to be less reliable sources of information than perception and inference: beliefs grounded on memory and testimony are seemingly more prone to falsity than those acquired through immediate perception or inference. What is the impact of this difference in reliability on the question of whether memory and testimony can be considered as sources of legitimate knowledge? This section is devoted to the distinction between de facto reliability of a source of belief on the one hand, and one's warrants to rely on this source, on the other.

In order to make clear the difference between the elements that transform true belief into knowledge (warrants) and the actual reliability of a potential source of knowledge, we first come back to perception and inference. Although immediacy seems to be a good barrier against error, perception and inference too can deceive and mislead us. The success of any knowledge-acquisition process based on them depends on the proper functioning of our cognitive (perceptual, inferential) faculties. However, their proper functioning, which is the background condition for their actual reliability in a given inquiry, is an empirical fact that is to be distinguished from the rationality of our epistemic practice of relying on these sources. In the absence of any recognized disfunction or of any particular reason to doubt one's competencies for a particular task, it is perfectly rational to rely on perception and inference. Given de facto reliability, perception and inference are legitimate sources of knowledge.

Memory also can lead us astray. As a result, the more one has to rely on it in a reasoning process, the more one is likely to make mistakes. But this is about de facto reliability, not about warrants. As for warrants, if I remember that $p$, and if I have no particular reason to suspect my memory to be mistaken, I am warranted in believing that $p$. Thus, as Burge argues, there is no major difference with reliance on perception and inference. 
Just as I cannot but rely on perception and reasoning (which are also fallible), I have to rely on memory. Accordingly, other things equal, it is rational to rely on it, even when I cannot access the reasons why it is so. To put it in other words, I am entitled to rely on memory even though I am unable to provide an explicit justificational argument for such a reliance (for the difference between entitlement and justification, see section 4, where this distinction is of central importance).

The same distinction and claim hold for testimony. Although one cannot be certain that testimony-based beliefs be free of errors, it is perfectly rational to rely on the word of others, just as it is rational to rely on one's senses, reasoning capacities, and memory (Burge 1993). On the one hand, if we, as epistemologists, cast doubt on the rationality of people's reliance on the word of others, we are to conclude that the vast majority of what people believe does not count as knowledge. On the other, Burge's point, which we endorse, goes further, ${ }^{2}$ as he argues that one's warrant to rely on testimony is a priori. ${ }^{3}$ Indeed, it "comes with [one's] being a rational agent" (Burge, 1993, 467). Other things equal (in the absence of reasonable doubt), we need no further justification to "accept as true something that is presented as true and that is intelligible to [us]" (ibid.), because "the seeming intelligibility of another's talk gives one apriori warrant to presume that the other is a resource for rationality", and resources for rationality are "other things equal, to be believed." (Burge, 1998, 5)

Thus, as rational agents, we are entitled to rely, other things equal, on perception, inference, memory and testimony. Again, this is not to deny that these sources can lead us

\footnotetext{
${ }^{2}$ Nobody, in fact, would claim that reliance on the word of others is irrational. The controversial question, which is at stake in the reductionism/anti-reductionism debate in the epistemology of testimony, rather concerns the nature of our warrant to rely on testimony. Reductionists, in the line of Hume (1740), argue that our epistemic right to trust others is derived from, and reducible to, our epistemic right to rely on observation and induction (roughly, we can trust others because we have observed that they are generally trustworthy). For antireductionists, whose classical figure is Reid (1764), reliance on testimony is a primary, fundamental, epistemic right.

${ }^{3}$ This also applies to memory, but it is more controversial in the case of testimony (although reductionism about memory has some advocates, such as Chisholm, 1966).
} 
astray. We may have empirical reasons to reject their offerings. But, in the absence of doubt, "rational acceptance of another's word", just like reliance on perception, inference, and memory, "is an apriori default position" (Burge, 1998, 5). The reliability of our sources, which is an empirical fact, constitutes the background condition (Burge, 1993, 463) of the success of our process of knowledge-acquisition. But our empirical knowledge of this reliability does not have to be invoked in the justification of the beliefs so acquired. This empirical element is not part of our warrant, which is a priori.

As our inquiry into the warrants of computer-based knowledge will rely on the distinction between a priori and empirical warrants, we shall now make clear in more detail the significance of the a priori character of our warrants to rely on memory and testimony. Warrants' being a priori is directly related to the fact that memory and testimony are contentpreservative: when they function correctly, they just supply information without altering it. They are, so to speak, "transparent" conveyers of information:

[Memory] supplies the propositions that serve as links in the demonstration itself. Or rather, it preserves them, together with their judgmental force, and makes them available for use at later times. One does not justify the demonstration by appeals to memory. One justifies it by appeals to the steps and the inferential transitions of the demonstration.” (Burge, 1993, 462). ${ }^{4}$

If I remember that $p$, I am warranted in accepting $p$, without having to invoke my experience of remembering that $p$ in the justification. Since "memory and perception of utterances function similarly, in reasoning and communication respectively" (481), this applies to testimony too. Even though testimony goes through auditory perception (in the simplest

4 To be sure, memory sometimes plays a different role in our mental life, and it can sometimes serve as an independent element in justification: "Memory of events, objects, experiences, or attitudes may form a premise in a justification of an empirical belief" (Burge, 1993, 464). In this case, it is better to talk about "substantive" memory, in order to distinguish this epistemic role from the former, purely preservative one. 
cases), the empirical fact of one's being told that $p$ does not have to feature in the justification of $p .^{5}$ The content of the knowledge is not altered by its being acquired through a process relying on memory, or testimony.

As a consequence, acquiring mathematical knowledge through memory or testimony does not ipso facto make the pieces of knowledge so obtained empirical. It remains a priori, whenever its justification (before its being transmitted through memory or testimony) was itself a priori. Of course, knowledge whose justification rests on memory or testimony can also be empirical; but it is so because the content that has been stored in memory, or that is being transmitted by testimony, has itself an empirical justification (because, at some point in the chain of warrants, some perceptual experience of our own, or of our interlocutor, was necessary to justify it), not because of its being acquired through memory-based reasoning, or by testimony.

\subsection{From minds to computers}

Let us now turn to computer-based knowledge, focusing on computer-assisted mathematical proofs, such as the proof of the Four-Color Theorem. An important epistemological problem, here, is that humans cannot follow (and hence, check) each step of the computational process leading to the proof of the investigated mathematical proposition. The first question is thus whether, and to what extent, it is rational to accept the theorem, despite this loss of epistemic control. To answer this question, we need to know whether

\footnotetext{
${ }^{5}$ It is worth highlighting the distinction between two different kinds of empirical facts about memory and testimony that might appear in one's justification (but, according to Burge, do not). The first one is one's empirical reasons to consider the source as reliable ("I have observed that my memory has most often provided me with reliable information"). This is the central point of reductionism (see note 2), and the most discussed. The other one is one's present introspective experience of one's remembering, or one's auditory experience of being told something ("I happen to remember that $p$ ", "this person is telling me that $p$ "). Burge's conception of a priori warrants provides an answer to the two issues together. However, they are in principle independent and should not be mixed up, as they sometimes are.
} 
computers are reliable information processors and do not alter the input content in any unexpected or uncontrolled way, at least in the kind of cases under study. This is a factual issue (Is the electronic makeup of the computer correct? Is the programming well-designed?). In fact, we often do have very good empirical reasons to rely on the computational processes run on machines: for many tasks, they are much more reliable (and efficient) sources of information than our own cognitive systems. Hence, when certain conditions obtain (when their basic functioning is granted), computers can be considered as sources of knowledge, and scientists' reliance on them is warranted.

The second question is: What is the nature of this warrant? Hence, what is the nature of the knowledge so obtained? Is it genuine mathematical knowledge, or is its nature somehow altered by its being obtained by relying on the outputs of an external device whose operations are epistemically opaque to us? At first sight, one might be reluctant to admit that the epistemic status of a theorem, which no human agent is able to prove by her own reasoning abilities, is the same as a more traditionally demonstrated theorem. For example, Tymozcko (1979) and Kripke (1980) have both argued that the knowledge resulting from such theorems is not purely mathematical, since empirical elements (such as the laws of physics, and empirical facts about the construction of the machine) enter its justification. Burge (1998) argues that such views are mistaken: he claims that computers can yield genuine, unaltered, mathematical knowledge. His argument relies on an extension of his analysis of the epistemic status of memory and testimony to the case of computers: just like memory and testimony, computers (provided they function correctly) are content-preservative sources of knowledge. We present the main elements of Burge's arguments in the remaining of this section.

According to Burge, empirical facts about the physical reliability of the computer can increase or decrease our confidence in the computer's outputs: "we may and do support our belief with empirical information about the machine." (Burge 1998, 6-7) But, once the 
computer's reliability is established, its functioning properly is just a background condition that does not enter the justification, exactly like the proper functioning of our own brain, or the reliability of others. As a consequence, there are no more reasons, as far as epistemic warrants are concerned, to consider that the use of a computer transforms mathematical knowledge into empirical knowledge, than to consider that our brains (which are also material) perform any similar transformation.

One might object that there is a major difference between computers on the one hand and one's own cognitive abilities (including memory) on the other hand. Computers are external devices. When we prove a theorem by relying on our own abilities, we can check every step, hence we do have the proof, whereas in the case of computer-assisted proof, "[we] rely on the computer's having carried out the details. So the justification might appear to have to make reference to the computer's physical reliability" (Burge, 1998, 7). But this objection seems to be easily answerable, given Burge's analysis of testimony: others are also external sources of information, whose mental operations are inaccessible to us. Things are not so simple, though: others are rational sources, which computers are not, as they are not autonomous thinkers. They just operate on symbols, whose meaning comes from rational agents' interpreting them. This is precisely why reliance on the outputs of computers can be analyzed as an extension of reliance on testimony. Here too, "we have rationally intelligible and credible mathematical presentations from the machine"; hence "we can presume that we are being confronted with a resource for rationality, in the absence of specific reasons for doubt." (Burge 1998, 9).

In fact, Burge argues that the possible sources of errors that could cast some doubt on a computer-based mathematical proof either derive from human errors, or are similar to the kind of doubt one could cast onto the functioning of a human being. Sources of errors are mostly of two types: (i) defects in the physical makeup of the machine, and (ii) errors of 
programming. As for (i), assuming that the computer is properly built amounts to trusting the people involved in the material fabrication. Once it is granted that the machine is correctly built, this empirical fact is not part of the warrant. As for (ii), Burge argues that, from an epistemological point of view, errors in programming are analogous to errors of expression in ordinary mathematical argumentation. Hence, trusting the basic programming of the computer amounts to trusting the programmers. One can thus consider that what the computers tell us come from others, and one can consider computers as rational sources:

The meaning of the machine's activities, and indeed the nature of its rational powers, are derivative from intentionality and rationality of rational beings. Such activities provide an amplification of the designers' and programmers' rationality. In understanding the machine's offerings, we have an apriori entitlement to rely on the rationality of these offerings, and the justifications backing these offerings. (Burge, 1998, 9-10)

It is worth mentioning another difficulty with the extension of the analysis of testimony to the analysis of computers. In the case of computer-assisted proofs, the result is a difficult one. In contrast with ordinary communication, we do have reasons to doubt, and acceptance might not be the rational, default, attitude. But this is not different, as Burge emphasizes, from cases of communication of a difficult mathematical result by someone else. In such cases, one does need a "supplementary justification to maintain acceptance" (Burge, 1998, 13), such as reasons to attribute specific competencies to our interlocutor, which make him trustworthy in the case at hand. Hence, this problem is reducible to the problem of the attribution of expertise, which we will come back to in section 4. For the moment, let us just note that, once we have grounds to maintain acceptance, our warrant to rely on the word of 
others - hence on computers - remains a priori. ${ }^{6}$ Empirical facts about the specific credentials of one's interlocutor do not need to enter the justification.

It is also worth noting that it is straightforward to extend Burge's analysis to the case of numerical experimentation, that is, the numerical exploration of equations (typically nonlinear ones) that do not have analytic solutions. Even though numerical experimentation has something experimental in it, and even though it is processed on concrete machines, the point is that nothing in the justification of the obtained results relies on perceptual interaction. Interactions with the computer, which are an essential part of the "experimentation", belong to the background conditions of the epistemic process but should not be included in the arguments by which the truth of the result is justified.

2.4 Taking stock (1)

${ }^{6}$ Burge (1998) shows that the different kinds of reasons one might have to "maintain acceptance" can all be analyzed as being grounded on our own reasoning abilities - hence that none of them transforms our warrant into an empirical one. 
Where are we now, and what does the analysis of computer-assisted mathematical proofs contribute to our study of the empirical warrants of computer-based knowledge? The first conclusion we can draw is that, in the case studied by Burge (1998), reliance on computers is warranted, this warrant being a priori. Once their correct functioning is granted, computers can yield genuine mathematical knowledge, without the content of this knowledge being altered. This would also apply to computer-based empirical knowledge, would computation proceed from a given set of initial premises without any further elements added during the computational process (see below). The important point is that the computational process does not, as such, disrupt the chain of epistemic warrants, and, consequently, that the content of the knowledge being processed is preserved. Hence, we shall assume that, in the cases, in which one can show that reliance on computers is warranted (and this might be a difficult, case-by-case, affair), the fact that knowledge is acquired through a computational process does not, by itself, alter the nature (empirical or a priori) of the piece of knowledge under consideration. This will be a crucial assumption for the rest of our analysis. In the case analyzed by Burge, the content being preserved by the computational process means that the resulting knowledge remains a priori, hence is genuine mathematical knowledge. As for empirical knowledge, content preservation means that, if we could show that all the empirical elements in a computer simulation are empirically warranted independently of the computational process (that is, before their being processed by the computer), then we could conclude that the resulting knowledge is empirically warranted. Obviously, as soon as there are empirical elements in the chain of warrants, knowledge is empirical. The issue we want to clarify is rather in virtue of what kind of empirical warrants knowledge obtained through computer simulation is genuine knowledge about the system under investigation. If the only warrant we could find out was that a computer is an object in the world, we would only be left with a pseudo-empirical, unsatisfactory reason for relying on simulations. From the above 
analysis, we have ruled out this option. Now, we want to know whether there are any good empirical reasons to accept the outputs of computer simulation as providing knowledge about the systems we are interested in. As will become clear, the demand for good empirical reasons implies that, at some point of the chain of warrants, some reference be made to the system under investigation.

Our task, now, is thus to clarify the origin of the empirical elements entering into computer simulations, so as to establish the conditions for their justification.

\section{Can computer simulations yield empirically justified knowledge?}

In this section, we address the first problem we have identified in the introduction (P1): as the computational process does not preserve the content of the theoretical model applied to input data, the question arises of the origin and justification of the elements that enter the process but that belong neither to the original, theoretical model ${ }^{7}$ nor to the input data. In order to make clear why (P1) threatens the validity of computer-based empirical knowledge and how this threat can be met, we first need to characterize the involved computational process in more details. This is why we will start by discussing two opposite (mis)conceptions about computer simulations and their value as empirical knowledge-providers that entail tentative answers to (P1). Discussing these conceptions will help us argue in favor of our own answer to $(\mathrm{P} 1)$ by contrasting it with the other answers. The first conception we shall discuss is a highly idealized view, according to which the computational process can be considered content-preservative; from this perspective, computer simulations do not add anything to the empirical content of the original model and input data and the empirical warrants of the

7 Here, we use "theoretical model" only to refer to the set of equations that are subsequently transformed into lines of code, and not to contrast "theoretical" with, e.g., "phenomenological". 
outputs are the same as the empirical warrants of the theoretical model and the input data (section 3.1). In section 3.2, we briefly present the view according to which the fact that the simulation is run on a material machine is part of why its results are empirical, and empirically justified. These two conceptions are misleading, and they obscure what we take as the real warrants for the empirical elements of computer simulations, which we present in section 3.3.

\subsection{An idealized view of computer simulation}

It is well-known to every user of computer simulations that there is hardly anything as messy as a simulation program, for bugs and errors are steadily lurking, and fine-tuning is indispensable over and over again. However, from an outsider's point of view, it might be tempting to abstract from these practical considerations and to conceive of the computational process in a detached way as a deductive one applied to an initial set of input values for variables and parameters in a given set of equations. After all, the aim of the simulation program is to implement mathematical transformations of the original model's equations in order to find out their solutions for particular sets of input values. The view here discussed is that idealizing away from the (otherwise recognized) non strictly content-preserving aspects of the computational process results in a fairly good approximation. Thus, according to this view, the computational process does not add anything important to the description of the system's initial state that is summarized in the set of the variables' initial values. In other words, the computational process does not alter the empirical content of the theoretical model applied to input data: if both correctly describe the initial state of the investigated system, then the outputs do so as well. The outputs are thus empirically warranted to the exact extent that the set of initial values and theoretical model are. However, the simplifications involved 
in such an idealized view of computer simulation are much too crude. Emphasizing why this is so makes clear why this idealized answer to (P1) is not satisfactory.

Before entering into our critical discussion of the idealized view we have just presented, we have to mention that some rare cases of simulations comply with it. This happens when the computer model is analytically solvable ${ }^{8}$, in which case it is possible to control the correctness of the outputs, if not the fine-grained details of the computational process, and thus to provide full-fledged justifications for the outputs. However, these cases do not raise any specific epistemic difficulty. This is the reason why we now turn to more common cases in which computer models are used to solve intractable equations.

Then we have to make clear that in this paper, we restrict ourselves to simulations based on the discretization of differential equations, leaving other cases for another paper. The most important point here is that in general, a discretized differential equation, whatever the discretization method employed, is not strictly equivalent to the original differential equation, from a mathematical point of view. That is, in contrast with the case studied by Burge (1998), the mathematical content of the original, differential equation is not exactly preserved, or left unchanged, when it is transformed into a finite difference equation. As a consequence, the empirical content cannot be considered as unchanged. Let us make this point more explicit. Usually, the empirical adequacy of a theoretical model is evaluated in its original form: what is taken into account in this evaluation is the set of continuous equations. Moreover, what is called "input data" is usually obtained from results of measurement. However, the original equations and measurement results are not the object of the computation. Adjustments have to be made which transform both the mathematical content and the empirical content ${ }^{9}$ of the

\footnotetext{
8 We thank an anonymous reviewer for asking us to mention these cases in order to complete our analysis of computer simulations.

${ }^{9}$ It is sometimes difficult to distinguish between mathematical and empirical content; however, this distinction is not so important for our argument as we focus on the empirical warrants of content transformation (be the content mathematical or empirical).
} 
theoretical model and input data. For instance, approximations (which imply transformations of numerical values) and simplifications (which imply transformations of what is said about the behavior of the investigated system) have to be made in order for the computation to run (cf Winsberg 1999). These transformations usually jeopardize the empirical adequacy of the original model and input data. In other cases, tuning techniques have the opposite result in improving the fit between the simulation outputs and the properties of the investigated system. However, we are not mainly interested in these cases as we focus on situations in which the original model being judged empirically adequate, the transformations it undergoes in the computational process threatens the empirical adequacy of its outputs.

It may be replied to this point that there are well-developed mathematical methods whose aim is to guarantee that the discrete equation resulting from discretization techniques is as close as possible to the original, continuous one. We have two counter-objections against this argument. First, discretization procedures only induce a small part of the transformations we are dealing with, as in some cases, even discrete equations are impossible to solve numerically. Second, in the context of the analysis of warrants we put forward in this paper, there are only two possibilities: either the computational process is content-preservative throughout, or it is not; were there only one addition or transformation in it, the details of the justification procedure of the simulation outputs would be different. If the computational process is content-preservative, the empirical justification of the outputs lies in the care with which the initial values have been determined and the original model designed; if it is not, the question remains open whether, and how, the outputs are justified.

Let us discuss this point further. It might be the case that the differences between the actually carried out calculation (which is not strictly content-preservative) and the intended one (which is meant to be content-preservative) are not epistemologically relevant: maybe the computational process, usually involving so much approximations and various algorithmic 
tricks, is almost preserving the content of the original equations. The above-mentioned mathematical theorems aim at suppressing epistemologically relevant differences, after all. However, as already emphasized, many simulations go beyond the applicability conditions of these theorems. Accordingly, no general claim can be made; it is all a very difficult case-bycase affair.

The upshot of our criticism of the exaggeratedly idealistic view of computer simulation we have presented in this section is that it is erroneous to claim that a computer simulation draws its empirical content from the empirical content of the original model and the initial values assigned to its variables and parameters alone. Many other elements from empirical origin force their way into the process at virtually every step of the program writing and checking.

\subsection{The materiality thesis}

Now that we have presented some reasons why the view that a computer simulation is reducible to a pure calculation process has to be rejected, we have to face the opposite view, according to which the fact that the simulation is run on a material machine is part of why its results are empirical. This claim is currently known as the "materiality thesis". Its main motivation is that, according to Parker, "inferences about [...] target systems will take as their starting point the observed behavior of a material system - the programmed digital computer — and accounts of the epistemology of computer simulation should reflect this." (Parker, 2009, 491) This claim can be understood in two ways. According to the first, the material dimension of computer simulations (the fact that they are run on material machine), and especially the potential malfunctioning of the machine, are relevant to assessing whether the results are warranted. We do endorse this first way to understand the claim, as must be clear 
from the rest of the paper. On its second interpretation, the materiality thesis says that simulation results are empirical data about the target system because the simulation is run on a machine ${ }^{10}$. Let us now present our objection to this view.

The defenders of the materiality thesis in its second form has it that the computer, as a material object, is the place within which an experiment is performed, mimicking the target experiment on the physical system under investigation, and the screen the place where its results can be observed. Because the computer, informed by an adequate program, shares some relevant, structural properties with the target system, the experiment performed on the computer provides the observer with reliable, empirical information about the target system. The empirical warrants of the simulations' results thus derive from the computer program being run on a (correctly programmed) material machine. Thus the solution to (P1), according to this view, lies in the structural similarity between the behavior of the investigated system and the computational process that runs on the machine.

As the materiality thesis has already been discussed, we will be content with recalling the main criticism it has received, due to Humphreys (Paris 2010 talk): "in digital computer simulations, the material implementation is relevant only in providing a concrete, temporally dynamic basis, a feature that is common to all digital computer simulations and [...] what is central to such simulations is the computational template that serves as a key element of the underlying computational model." Humphreys insists that the computer's materiality is secondary with respect to the computational model and that it does not have to enter the assessment of the simulation's results. For sure, the calculation being implemented on a material machine is a necessary condition for the process yielding the expected results to occur, but it is not necessary for the interpretation of the results as being about the target system. Therefore, the materiality thesis, which implies that the materiality of the machine is

\footnotetext{
10 We thank an anonymous reviewer from pointing out these two forms to us.
} 
responsible for the empirical character of the simulation's results, is as erroneous as the idealized view sketched in section 3.1. The empirical justification of a simulation's outputs is fully independent from its being run on a material machine.

We are now left with two negative results:

- the empirical elements in a computer simulation are not restricted to the empirical content of the original model and initial values of the program's variables and parameters;

- the empirical justification of the simulation's outputs has hardly anything to do with the simulation being run on a material machine.

This does not tell us much about the way the simulation outputs can be empirically justified. However, Humphreys' criticism of the materiality thesis points towards a more constructive answer: he emphasizes that the computational model is responsible for the simulation being relevant to this target system, as opposed to any other one. Following his suggestion, we investigate in the next section in what sense the computational model gives the computer simulation the power to bear an empirically justified content.

3.3 Interpreting the simulation's outputs as being about the investigated systems

In AUTHOR et al. (2009), one of us has argued that the reason why computer simulations may provide us with valuable information about target systems is that they are intentional artifacts whose semantic purpose is precisely to represent the behavior of the target system, hence to extract as much information as possible from the theoretical models they rely on, when applied to the target system. Whereas the claim that simulations are intentional artifacts is uncontroversial, its implications are not. Let us review some of them, with a special emphasis on those related to the empirical justification of the simulation outputs. 
The first task is to clarify what a computer simulation consists of. Are we talking about the computer program as a set of lines of code, or about one run of the program, or else about a series of runs on which statistics are performed? The following options are to be distinguished (see AUTHOR et al., 2009, 566-567):

(a) an incomplete program that includes every single algorithm that is actually used (to be completed, this program only requires that someone enter the initial values of the relevant variables);

(b) a completed program that only requires someone to press the "run" key to run and to provide outputs;

(c) the running completed program, a process corresponding to the exact running of (b);

(d) the data resulting from (c), that is, the simulation outputs;

(e) the computer-made analysis of the large sets of data (d).

Whereas strictly speaking, the simulation outputs are those described in (d), the scientists running the simulation are only interested in (e), from which they may infer important information about the target system. For the simulation to fulfill its representational function, it is necessary that the various parts of program (b) that are devoted to the simulation proper be carefully written and checked. The other elements in (b) include various operations such as the writing of numbers in a folder, test operations, the opening of interfaces for visualization, etc. These operations do not have any representational role. The simulating parts of (b) compute the mathematical functions whose results are interpreted as giving empirical information about the target system.

The outlined description of the simulation program and its outputs shows that if a computer simulation is an intentional artifact, it is nevertheless difficult to exactly tell what is supposed to bear intentional content within it, and a fortiori empirical content, let alone 
empirically justified content. Further analysis reveals that the running completed program (c) can be understood in at least four different ways (AUTHOR et al., 2009, 568):

(1) as a succession of physical states of the machine on which the program is run;

(2) as a succession of computational states of the machine;

(3.1) as successive sets of values for mathematical, discrete iterative functions;

(3.2) in some cases, as successive sets of values of solutions of differential equations taken at regular intervals;

(4) as successive numerical representations of physical states of a physical system governed by laws.

The intended interpretation in any normal use of a simulation is (4); however, (4) is the final result of the preceding interpretative steps, so that the running program can only be understood as (4) if the preceding interpretative steps are free of errors.

The above analysis allows us to determine the general conditions under which a computer simulation is susceptible of yielding the expected, empirically justified results. The first condition is that the machine correctly function, so that the succession of its physical states can be interpreted as a succession of computational states on a regular basis. The fulfillment of this condition is evenly presupposed by the scientists interested in the outputs (e), even though, in principle, it should not be taken for granted. But the epistemological problem raised by the question of the machine's physical reliability is the same for computer simulations as for computer-assisted mathematical proofs (see section 2.3). Second, the differential equations of the original model have to be discretized so as to guarantee that their solutions do not diverge from the solutions of the continuous equations. This condition is always difficult to fulfill and has to be checked on a case-by-case basis. Third (but this is the first condition from the practical point of view), the differential equations that are supposed to govern the behavior of the target systems are (at least approximately) the right ones. Each of 
these conditions can be further decomposed into more detailed ones, depending on the specificities of the studied computation. In order for the simulation outputs to be justified, all of the above general conditions have to be satisfied, and the initial values and parameters have to be themselves empirically justified.

\subsection{Taking stock (2)}

So far, we have discussed three conceptions of the conditions under which we can obtain empirically justified knowledge from a computer simulation. Contrary to what the first two (sections 3.1 and 3.2) suggest, the upshot of our discussion is that it is hardly possible to provide a full-fledged argument showing that the obtained information is empirically justified. The capacity of the whole process to yield empirically justified outputs depends on many different elements, such as the quality of several algorithms one might have borrowed from a specialized website, the adequacy of the theoretical model, etc. In principle, none of these elements should be taken for granted if one were to give a full-fledged, unobjectionable, justification of one's acceptance of the result. However, in practice, the scientist using the simulation in order to investigate an empirical phenomenon never has full control on all these elements. Therefore, individual agents cannot possibly access any stricto sensu, full-fledged, justification for their building up empirical knowledge from simulation outputs. This is the reason why it is hardly possible to provide an explicit, complete argument showing why it is rational to to rely on computer simulations.

It is thus necessary to switch from a general inquiry into the conditions for a fullfledged, in principle, justification of the empirical knowledge drawn from computer simulations (which agents can never access), to an inquiry into individual scientists' entitlements, in context. We shall thus investigate when, and in what conditions, scientists are 
rationally warranted in assuming that the various above-mentioned conditions for the capacity of the process to yield empirically justified outputs (quality of algorithms, etc.) obtain. As we will see, this implies a shift in the focus of our inquiry. As the scientists' minds are the place where epistemic warrants emerge when they track the transformations of empirical content during the computational process and assess whether the result is warranted enough, we will have to adopt the agents' perspective, taking into account their background knowledge, and their epistemic goal in a given inquiry. We shall thus claim that despite poor epistemic access to the details of the computation, individual agents are crucial components in the building up of empirical warrants for the results of computer simulations. Accordingly, we now move to the analysis of the way warrant flows through individual agents.

\section{To what extent are agents warranted in accepting empirical knowledge from computer simulations?}

In section 3, we have shown that the justification of the results of a computer simulation cannot come from any general analysis but rather depends on the specificities of each case. As a result, a full-blown epistemological analysis of computer-based knowledge has to involve other elements than those traditionally taken into account in the practice of justification. To put it in a nutshell, such an analysis cannot be restricted to the elements that can be explicitly stated, like reference to such or such general principle or to such or such mathematical theorem, but has to extend to more implicit elements, like what individual agents take for granted but can hardly express in a public fashion. In this section, we thus examine the specific conditions the individual scientists face when working with computer simulations, in order to uncover what, in terms of entitlements, they rely upon in accepting the simulations outputs. 
We first (4.1) present the impact of (i) epistemic opacity (the scientist' inability to follow the calculations) and (ii) the division of scientific labor (her reliance on the work of many colleagues who have taken part in the elaboration of the computer program) on the retrieval of empirical knowledge from computer simulations. We then (4.2) analyze the reasons why scientists, despite these epistemological difficulties, are rationally warranted to rely on the simulations' outputs in their empirical investigation, when certain conditions obtain.

4.1 Working conditions of the simulation's user: Epistemic opacity and division of scientific labor

As mentioned in the introduction of this paper, scientists using computer simulations are unable to fully access, hence control and understand, the details of the computational process (P2). Humphreys $(2004,147-151)$ calls this phenomenon "epistemic opacity". In section 2, we have presented Burge's analysis of this problem, as it arises in the case of computer-aided mathematical proofs. Armed with our analysis of the empirical elements of computer simulations (section 3), we now have to explore the consequences of epistemic opacity in the case of the computer-based production of empirical knowledge. In this section, we aim at assessing the impact of epistemic opacity on the way scientists retrieve empirical information from computer simulations. In order to do so, we provide further details on the origin and impact of this phenomenon.

In order to analyze epistemic opacity, defined as the inability to follow the computational process, we first need to make clear which features of the computational process are relevant to this analysis. We shall see that some of these features can safely 
remain opaque whereas blindness to others is a threat to the rationality of one's acceptance of the results of computer simulations.

A computation involves a dynamic series of unit actions on content-bearing objects or symbols. In a computer simulation (based on a model involving differential equations), the symbols are of different kinds, so that different computations are performed at once (see section 3.3):

- The first one is only carried out with the help of 0 s and $1 \mathrm{~s}$; it is the most basic one, expressed in the unique language that is directly accessible to computers, so to speak. By contrast, this language of $0 \mathrm{~s}$ and $1 \mathrm{~s}$ is unintelligible to human beings (when more than a few lines are concerned). However, this is not the main cause of epistemic opacity for it is not related to the right aspect of the performed computation. Epistemic opacity has to do with the mathematically interpreted computational process, as designed to provide solutions to the original model's equations. As the dynamic series of $0 \mathrm{~s}$ and $1 \mathrm{~s}$ are only a means to obtain these solutions, being unable to follow them does not mean that one cannot follow the relevant mathematical calculations.

- Another type of computation is performed on integers. Here, "computation" is construed as a mathematical calculation upon integers. It is impossible to follow this computation in practice because (i) of the pace and huge number of arithmetical operations and (ii) of the numerous sub-programs that do not take part in the computation proper, but are necessary to control the smooth running of the computational process, for instance by avoiding divergences or the possibility that a denominator equals zero. These sub-programs are necessary for the computation to give correct results, but they are not part of the calculation upon integers, so that they are unnecessary to a proper understanding of this calculation. Correspondingly, whereas factor (ii) is a source of epistemic opacity, it is irrelevant to consider it when spelling 
out the epistemological significance of epistemic opacity, since it is not part of the intended calculation.

Factor (ii), however, has to be further analyzed in order to provide a fullfledged account of epistemic opacity. Pace and number of operations are not the only difference between calculations performed by computers and calculations performed by human beings. Whereas the computer proceeds by mechanically repeating the same series of simple operations a large number of times, humans are sometimes able to use different, less time consuming methods to solve differential equations. Human beings, even when using numerical methods, do generally not use such mechanical procedures all the way down. They appeal to ingenious short cuts, they look for easy ways to find intermediary results, etc. As for analytic solutions, they are found out in a completely different manner from the operations of the computer. It is thus clear that computers and human beings follow fairly different ways to find out solutions to differential equations, and even to discretized equations.

As a result, although human beings can in principle follow or understand what the computer does (that is, when pace and number of operations are neglected), such an understanding is abstract in the sense that it contributes nothing to the intellectual process humans have to carry out in order to complete the same task as the computer. This is the main reason why factor (ii) is a source of epistemic opacity.

- The last type of computation that is performed by the computer during the running of a simulation is the "intended" computation, the one physicists are interested in: the computation of the solutions of the model's differential equations. As it is not performed exactly, epistemic opacity in terms of pace and number of operations is accompanied by the impossibility of following all the approximations, idealizations and corresponding recipes aiming at guaranteeing that the computation is well-behaved, namely that the 
outputs are plausible and that the computation does not stop at some point (these aspects are analyzed by Winsberg 1999).

As appears in the preceding analysis, epistemic opacity is not always threatening the justification status of the results of computer simulations. However, when it bears on the "intended" computation, scientists lack the traditional warrant against error consisting in checking out every elementary operation in the calculation chain. As a consequence, they are usually unable to provide their colleagues with full-fledged justifications for the results they draw from computer simulations, in the form of valid, or at least correct arguments explicitly expressed.

Epistemic opacity is not the only reason why individual scientists working with computer simulations are usually unable to design full-fledged justificational arguments about the validity of the empirical information they draw from the simulations. Most of the time, they also develop their projects on a collaborative basis. As a result, they have to rely on the skills and words of others. For instance, it is common practice to pick up sub-routines on specialized web sites without taking the time (or even being able) to check their efficiency or relevance, or the way they connect to the main program. The collaborative character of many computer simulations thus adds to the difficulty of grounding justificational arguments about their outputs.

At this point, the epistemic state of scientists aiming at retrieving empirical knowledge from the outputs of computer simulations might appear more dramatic than in the case of computer-assisted mathematical proofs studied by Burge. The scientists' partial blindness to the details of the computational process seem to result in a serious lack of epistemic control upon the empirical validity of its outputs, which might dangerously threaten the rationality of their reliance on these outputs. In the following section, we show that it is in fact possible to extend Burge's approach, and apply his epistemological categories, to the case of computer 
simulations. Whereas scientists cannot usually express in a fully explicit way the epistemic warrants they rely upon when accepting the results of computer simulations, they are not irrational for all that.

\subsection{Reasons to rely on the simulation's outputs}

Because of epistemic opacity and the division of cognitive labor, it is impossible for the user of a computer simulation to reconstruct, even approximately, full-fledged justification arguments for the validity of the simulation's outputs, that is, explicit arguments containing all relevant premises and ending up in the desired conclusion. However, we claim that she is not bound to blindly trust them, or to reject them altogether, for all that. In this section, we will clarify the conditions under which a scientist is rationally warranted to use the outputs of the simulation in her further investigations.

First (4.2.1), we emphasize, following Burge's distinction between different kinds of epistemic warrants, that an agent may be entitled to certain epistemic practices even though she is unable to explicitly express her rational grounds (Burge, 1993, 458-459), and we suggest how this claim can apply to the case of the use of simulation outputs. Then (4.2.2), we further explore this view by analyzing other cases in which scientists lack full-fledged justifications but are nevertheless entitled in accepting elements of knowledge they cannot fully control. Finally, we draw the conclusions of this analysis for the case of computer simulations (4.2.3).

4.2.1. The notion of epistemic entitlement 
Epistemic justification may come in a variety of ways. The most celebrated one is the way of full-fledged arguments, deductive or inductive, which are potentially public and fully explicit. In order to possess a full-fledged justification argument for some conclusion, one has to be able to explicitly express the premises and assess the validity (or the correctness) of the logical link between the premises and the conclusion. In other words, being fully justified in entertaining a belief consists in being able to articulate the whole chain of reasons leading to the belief.

Explicit arguments are only one type of epistemic warrant, however. A major distinction can be put forward between (full-fledged) justification and entitlement. ${ }^{11}$

Although both have positive force in rationally supporting a propositional attitude or cognitive practice, and in constituting an epistemic right to it, entitlements are epistemic rights or warrants that need not be understood by or even accessible to the subject. (Burge, 1993, 458)

In fact, our warrants to rely on perception, inference, memory and testimony, which we have discussed in section 2, are entitlements, rather than justifications (Burge, 1993, 458, 1998, 3). In most cases, our reliance on these sources is rational, even though we are not "able to justify [such] reliance [...], or even to conceive such a justification." (1993, 458-459) Whereas "justifications, in [the] narrow sense, involve reasons that individuals have and have access to" (Burge, 1998, 3), entitlements may be left opaque and partly inaccessible to the agent. "Children are entitled to rely on particular perceptual beliefs even though they could not understand why. Perhaps only philosophers can explain why." (ibid.) The notion of entitlement thus accounts for the commonly held intuition that many of our everyday epistemic practices are rational, and do yield warranted beliefs (and genuine knowledge),

11 The notion of epistemic entitlement has also been discussed, among others, by Dretske (2004) and Wright (2004). Here, we stick to Burge's conception. 
without our being able to articulate why they are, let alone to devise any sufficient argument in favor of these beliefs.

From this perspective, the question arises whether, and in what conditions, a scientist may be entitled to accept the outputs of a simulation and to rely thereon in her further investigation even though she may not know how to possibly justify them by rigorous arguments, as she may not know the rational grounds on which they are based. An important characteristic of entitlements in an inquiry of this type is that they are context, and agentdependent. Indeed, as emphasized by Burge, the notion of entitlement "applies not primarily to representations or representational contents independently of some representer. It applies to individuals' representational faculties, abilities, commitments, practices, states and events." (Burge, 2003, 506) The warranting force of entitlements depends, for each agent, on her specific set of beliefs and acceptances, as well as on her specific epistemic goal. The notion of entitlement is

conceptually tied to considerations regarding limitations of perspective, information, and structure in the particular system or animal at hand. Whether an epistemic state is warranted depends partly on the epistemic perspective of the individual in that state - what information the individual has or what information is accessible, and how well that information is used. (Burge, 2003, 506)

The clerk at the supermarket is certainly entitled to rely on the additions made by the till, without checking that the machine performs the operation reliably. Given his goal, his competencies, and the constraints of rapidity and efficiency, he is perfectly rational not to check, even once, if the machine functions properly, before charging the customer the sum 
total indicated by the till. ${ }^{12}$ This feature of entitlements makes the notion especially relevant to the analysis of scientists' epistemic attitudes towards the outputs of computer simulations. Indeed, scientists from different disciplines may be more or less reluctant to accept the outputs of a given simulation in certain circumstances. For instance, the specialist of numerical analysis may doubt the validity of the computed solutions of the initial equation because she knows more about the general behavior of the solutions, whereas the physicists using the equation in her representation of the investigated phenomenon may be content with the computed solutions. No two scientists rely on the same epistemic warrants, because each of them has progressively built up a (fuzzy) set of what she may rationally accept during her training and professional life. This feature not only influences the way scientists rely on a simulation's outputs, but also many other aspects of contemporary science, particularly when it relies on some division of cognitive labor.

The relative privacy of the scientists' set of acceptable epistemic warrants is no argument in favor of their irrationality. Therefore, by investigating scientists' epistemic attitudes, we do not leave the realms of reasons, but rather focus on reasons that are sometimes uneasy to put in words.

The question may arise whether appealing to reasons that are difficult to spell out is not a way to hide a purely pragmatic account behind a self-claimed epistemological analysis (we thank one anonymous reviewer for asking this question). Here, a purely pragmatic account is one which answers the question whether computer-based knowledge is warranted

\footnotetext{
12 We thank an anonymous reviewer for suggesting the example of the clerk. It shows the epistemological problems raised by epistemic opacity are dependent on the context, and on the agent's goal. The goal of the clerk might be described as collecting the amount that is indicated by the till (hence, his epistemic goal reduces to know what one can read on the machine), whereas the goal of the store manager might be to collect the amount of the goods the buyer actually has in his basket, which implies making sure that the machine does function properly. Now, the store manager himself might be entitled to rely on the tills company. Although epistemic goals, here, are subordinate to other practical goals (collecting money), this case provides us with a good analogy. In purely epistemological terms, and even assuming that the clerk's goal is to know what the sum of the values he has entered into the till amounts to, he can easily be shown to be entitled to rely on the machine's outputs.
} 
by only relying on the fact that scientists actually do as if it was, and do that for purely pragmatic reasons. That is, they do not have well-grounded reasons to accept the outputs of computer simulations but go on in their inquiry if they look plausible. We oppose such a pragmatic view and try to make explicit on which epistemic mechanisms confidence in the simulation outputs rely.

In order to do so, we will now discuss two other examples of epistemic attitudes in which applying the notion of entitlement is epistemologically fruitful: expertise, and reliance on "black boxes". In the last section, we will draw the consequences of this analysis for computer simulations.

4.2.2 Examples: expertise and "black boxes"

An expert is someone whom we acknowledge as more reliable than ourselves for some epistemic enterprise. The expertise relationship is based on the fact that some people have justifications (relative to a particular issue) whereas others only have entitlements (relative to this issue): we feel confident in the expert's judgement even though we are unable to access the exact reasons why it is justified (see Hardwig 1985).

Expertise shapes the relationships both between scientists and laymen, and among scientists of different disciplines. For instance, physicists usually rely on mathematicians, who better master the details of mathematical proofs. Even when physicists do not manage to follow every step of a mathematical proof, they are usually entitled to rely on its conclusion in their own investigations when a mathematician tells them the proof is correct.

When $A$ recognizes $B$ as an expert and $B$ claims that $p, A$ is entitled not to investigate into the reasons why $p$ is justified and to rely on $B$ 's judgement. In this case, $A$ does not need 
to open the box of the justifications of $p$, so to speak. Reliance on experts is thus an epistemic attitude that is close to the widespread practice of "black boxing". Here, we call "black boxing" an epistemic process of the following kind: let $B B$ be a supposedly reliable knowledge-producing device; if $A$ uses $B B$ 's outcomes without caring about how it works, she is black boxing $B B$, i.e., she does not exercise her epistemic authority on the way the outcomes are generated. Black boxing is practiced in the lab as often as in everyday life. Some observation instruments are so sophisticated, and at the same time so reliable, that it would be a loss of time to check the details of their functioning at each operation. Black boxing is a useful epistemic habit when the knowledge-producing device is reliable: it saves time and mental energy.

Both in the case of expertise and black boxing, the agent feels entitled to rely on the epistemic authority of someone else (or something) without being able to fully make explicit why she does so because of epistemic opacity or cognitive inaccessibility. In both cases, reliability is the main property allowing for renunciation to one's epistemic control. Moreover, once reliability is granted, one's entitlement to rely on such external sources as experts or observational instruments is a priori, meaning that the content of the knowledge is unaltered by its being acquired through these sources (see section 2).

Note that in the cases we have just mentioned, reliability has to be quick to assess. Because it is so important in academic life to be able to quickly decide to whom one can grant one's trust, scientists are trained to find out who are the reliable experts in their domains. As for observation instruments, establishing their reliability is just part and parcel of using them correctly. We do not mean that assessing the reliability of experts or of observation instruments is an easy matter; however, we emphasize that it is a pre-condition of modern scientific practice. As we shall see in the next section, assessing the reliability of a computer simulation is a similar, though more complicated, enterprise. 


\subsubsection{Computers}

As put forward by Burge, mathematicians are entitled to rely on the computational process taking place in the computer in the case of well-controlled computer-assisted mathematical proofs; the resulting knowledge is genuinely mathematical. Before investigating whether this attitude can be extended to the case of computer simulation, we first emphasize that judging oneself as entitled to rely on a computer-assisted mathematical proof requires a considerable amount of checking of the various sub-parts of the program that can be investigated by human beings independently of the remaining of the proof. It does not involve, by any means, blind trust.

Computer simulations also include mathematical calculations, which may be the object of entitlement. But they are meant to bear on empirical phenomena. Therefore, the question of whether one is entitled in relying on their outputs is different from the question as raised in the case of computer-assisted mathematical proofs. The simulation's outputs have not only to be interpreted as results of mathematical calculations, but also as bearing an empirical content, that is, as potentially providing empirical information about the system under investigation.

As we have emphasized in section 3, the interpretation of the simulation's outputs as containing information about the target empirical phenomenon is itself based on a series of interpretive steps, from the interpretation of the $0 \mathrm{~s}$ and $1 \mathrm{~s}$ forming the language of the computer, to the interpretation of the discrete mathematical formulae as representing continuous equations, and then to the interpretation of the formulae as representing the system's behavior. The first two steps are typically black boxed: the physicist is entitled to 
rely on the computer scientist, who has checked the functioning of the computer, in the same way as she is entitled to rely on the specialist of discrete mathematics, who has proved the (quasi)-equivalence between the original, continuous equation and its discrete transcription. So, black boxing, as analyzed above, is an important aspect of the epistemic relationship between the scientist and the simulation's outputs. In the same way, reliance on expertise is frequent. What about the third step (interpretation of the formulae as representing the system's behavior)?

As emphasized above, Burge claims that mathematicians are entitled to rely on computer-assisted proofs when they think (without being able to provide explicit justification for their conviction) that the mathematical content of the premises of the proof is preserved in the (mathematically interpreted) outcomes of the computational process. In the case of computer simulation, the content of the representation of the initial state of the system (as provided by the theoretical model and input data) is not strictly preserved throughout the computational process (pace the idealized view of computer simulations criticized in section 3.1). As a consequence, in order for the outcome to be a faithful representation of the system's final state (provided that the representation of the initial state is empirically adequate), the introduction of further elements of empirical origin has to be warranted. As we have seen in section 3, the individual scientist cannot check the origin of each of these elements: she has to rely on various programs, and algorithms provided by others, etc. Hence, the introduction of further elements into the computational process is empirically warranted provided that (i) others have good empirical reasons to use such algorithms and programs and (ii) that these reasons also hold for the empirical system at hand. Our claim is that this epistemic situation may be analyzed as an extension of the one we have analyzed in the preceding section when dealing with the division of scientific labor and trust in experts. 
In the same way as we interpret others' words as issuing from prima facie rational beings like us, but are epistemologically careful when accepting their testimony, scientists have to interpret the simulations' outputs as being about the investigated phenomenon, as they intend them to be, but they also have to be careful about possible errors and disfunction. The complexity of the epistemic attitudes we entertain when relying on others in order to get empirical information is transferred to the scientists' attitudes toward the simulation's outputs, and even augmented by the fact that the computational process is epistemically opaque. However, nothing, in principle, prevents scientists for being rationally entitled to accept the outputs and to rely upon them in further empirical investigations. Let us summarize our conclusions in terms of the two problems (P1) and (P2) presented in the introduction. $(\mathrm{P} 1)$ taken alone arises in the case of computer simulation just as in the case of computer-based mathematical proof, and it can be treated the same way. Our examination of (P2) has led us to conclude that the elements of empirical origin that are introduced in the course of the computational process are warranted insofar as they can be interpreted as bearing on the system under investigation. But scientists cannot check all this individually, among other things because of (P1). Our solution to (P2), given (P1), is that one can treat this case as an extension of scientists' reliance on experts: individual scientists have to trust others in the interpretive steps they have performed while designing their algorithms and proposing various idealizations and simplifications in the model.

The conclusion that scientists can be rationally entitled to accept the results of computer simulations might seem deceptive for it does not preclude that results are in fact accepted without good reasons. However, as our inquiry bears on matters of principle, we are content with showing that it is possible to find out reasons of accepting the results of a computer simulation. It seems to us that establishing this possibility is enough to answer our initial questions about the warrants of computer-based knowledge. Had it turned out that the 
reasons for accepting the empirical results of computer simulation were too elusive to be analyzed from an epistemological point of view, relying for instance on badly-defined capacities as "intuition" or "physical sense", the prospects of computer-based empirical knowledge would have been very bad indeed. As we have only examined the "easy" case, that is, computer simulations based on theoretical models that are supposed to be empirically adequate, although not solvable by analytical means, it would have been all the worst for the more difficult cases if we had found out that it was not possible to provide a rational account of one's reliance on simulation outputs.

Moreover, by relying on Burge's analysis of a priori warrants and content preservation, we have established that empirical knowledge drawn from computer simulations can be genuine empirical knowledge, meaning that its content is not altered by its being obtained through a computational process. When the introduction of empirical elements in the course of the computation is based on good empirical reasons, namely reasons that have to do with the system under investigation, there is no ground for being suspicious about the epistemic status of the outputs of the computation, even though scientists cannot individually access all the steps of the chain of reasons warranting these outputs..

\section{Conclusion}

A scientist wanting to rely on a simulation's outputs is usually aware of the inadequacies of the "motley methodology" which has been used in writing it up. However, in certain circumstances, she will be willing to rely on the simulation's outputs. We have tried to uncover what her reasons may be for doing so, taking for granted that she cannot access fullfledged justification arguments, and to clarify the nature of the knowledge so obtained. 
Any inquiry into the conditions in which agents are rational to rely on computer-based empirical information is conditioned by the fact of epistemic opacity: human beings are unable to follow what is going on within a computer simulation. As we have emphasized, this partial blindness has a major impact on the epistemic attitudes a simulation's user may entertain towards the simulation's outputs. Because of epistemic opacity, the epistemic warrants scientists explicitly or implicitly accept do not usually possess clarity or distinction. They are rather difficult to make explicit and precise. This is the reason why we have introduced entitlement as another notion of rational warrant besides the notion of justification when investigating the epistemology of computer simulation. This has enabled us to establish that, when certain conditions obtain, not only is it rational to rely on computer simulations, but the knowledge so acquired is genuine empirical knowledge, unaltered by its being obtained through a computational process.

\section{Acknowledgements}

We warmly thank the anonymous referees who allowed us to avoid mistakes and to enrich our paper.

\section{References}

AUTHOR et al. 2009. xxx.

Burge, T. 1993. Content preservation. The Philosophical Review, 102(4): 457-488

Burge, T. 1998. Computer Proof, Apriori Knowledge, and Other Minds. Noûs, 32, Supplement: Philosophical Perspectives, 12, Language, Mind, and Ontology: 1-37 
Burge, T. 2003. Perceptual Entitlement. Philosophy and Phenomenological Research, 67(3), 503-548.

Chisholm, R., 1966. Theory of Knowledge, 2d ed. 1977, 3rd edition 1989. Englewood Cliffs, N.J.: Prentice Hall.

Descartes, R. 1628. Rules for the Direction of the Mind, in Descartes, R., Philosophical Works, Haldane and Ross, eds., New York: Dover, 1955, vol. 1.

Dretske, F., 2000. Entitlement: Epistemic Rights without Epistemic Duties?. Philosophy and Phenomenological Research, 60 (3): 591-606.

Frigg, R. and Reiss. J., 2009. The philosophy of simulation: Hot new issues or same old stew? Synthese, 169 (3).

Hardwig, J., 1985. Epistemic dependence. The Journal of Philosophy, 82(7), 335-349.

Hartmann, S., 1996. The World as a Process: Simulations in the Natural and Social Sciences, in E. Heselmann et al. (eds), Modelling and Simulation in the Social Sciences from the Philosophy of Science Point of View, Theory and Decision Library. Dordrecht, Kluwer, $77-100$.

Hume, D., 1740. A Treatise of Human Nature, L. A. Selby-Bigge (ed.), second edition, revised P. H. Nidditch (ed.), Oxford: Oxford University Press, 1978.

Humphreys, P., 2009. The philosophical novelty of computer simulation methods. Synthese, $169(3), 615-626$.

Humphreys, Paul. 2004. Extending Ourselves: Computational Science, Empiricism, and Scientific Method, Oxford University Press.

Humphreys, Paul. Paris 2010 Talk. (Thought) Experiments and Simulations. Unpublished Manuscript.

Kripke, S. 1980. Naming and Necessity, Cambridge, Mass., Harvard University Press. 
Lloyd, E. 2010. "Confirmation and Robustness of Climate Models", Philosophy of Science, 77, 971-984

Lehnard, J., 2007. Computer Simulation: The Cooperation between Expertimenting and Modeling. Philosophy of Science, 74 (2), 176-194.

Morrison, M., 2009. Models, Measurement and Computer Simulations: The Changing Face of Experimentation, Philosophical Studies, 143 (1).

Parker, W., 2008a. Franklin, Holmes and the Epistemology of Computer Simulation. International Studies in the Philosophy of Science.

Parker, W., 2008b. Computer Simulation through an Error-Statistical Lens. Synthese.

Parker, Wendy. 2009. Does Matter Really Matter? Computer Simulations, Experiments and Materiality. Synthese, 169(3): 483-496

Reid, T., 1764. An Inquiry into the Human Mind on the Principles of Common Sense. In Inquiry and Essays, R. Beanblossom and K. Lehrer (eds.), Indianapolis: Hackett, 1983.

Rohrlich, F., 1991. Computer Simulation in the Physical Sciences. PSA: Proceedings of the Biennial Meeting of the Philosophy of Science Association 1990, 507-518.

Tymoczko, T. 1979. The Four-Color Problem and Its Philosophical Significance. The Journal of Philosophy, 76 (2), 57-83.

Winsberg, Eric. 1999. Sanctioning Models: The Epistemology of Simulation, Science in Context, 12(2): 275-292

Winsberg, E., 2003. Simulated Experiments: Methodology for a Virtual World. Philosophy of Science, 70 (1), 105-125.

Winsberg, E., 2009. A Tale of Two Methods. Synthese, 169(3): 575-592.

Wright, C., 2004. Warrant for Nothing (and Foundations for Free)?. Aristotelian Society Supplementary Volume, 78 (1):167-212. 